\title{
Measurement of Mean Arterial Pressure: Comparison of the Vasotrac Monitor With the Finger Differential Oscillometric Device
}

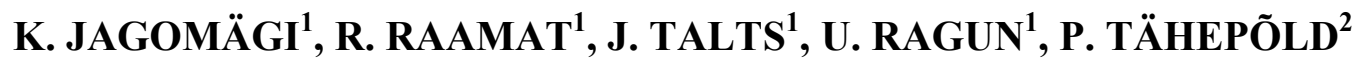 \\ ${ }^{1}$ Institute of Physiology, University of Tartu, Tartu, Estonia, ${ }^{2}$ Department of Anaesthesiology and \\ Intensive Care, Tartu University Hospital, Tartu, Estonia
}

Received April 16, 2009

Accepted January 29, 2010

On-line April 20, 2010

\begin{abstract}
Summary
The Vasotrac monitor provides non-invasive near-continuous blood pressure monitoring and is designed to be an alternative to direct intra-arterial blood pressure (BP) measurement. As compared to radial artery invasive BP and upper arm noninvasive BP, Vasotrac readings have been found to have a good agreement with them. However, discrepancies have been reported when rapid changes in BP exist. In the present study we compared BP measured by the Vasotrac monitor on the radial artery with that recorded on the finger arteries by the differential oscillometric device allowing measurement on the beat-to-beat basis. Comparisons were performed on the mean arterial pressure (MAP) level. Special attention was paid to the signal conditioning before comparison of pressures of different temporal resolution. Altogether 383 paired MAP measurements were made in 14 healthy subjects. Based on all 383 paired measurements, the MAP values measured at the radial artery at rest were $4.8 \pm 6.0 \mathrm{~mm} \mathrm{Hg}$ higher than those measured on fingers. The observed difference between the Vasotrac and differential oscillometric device can be explained by different measurement sites. This result is consistent with previous investigations, and the Vasotrac monitor can be considered to adequately track relatively rapid MAP changes on the radial artery. Attention should be paid to a proper signal conditioning before comparison of results obtained by different devices.
\end{abstract}

\section{Key words}

Vasotrac - Mean arterial pressure - Differential oscillometric blood pressure

\section{Corresponding author}

Kersti Jagomägi, Institute of Physiology, University of Tartu, 19 Ravila St., 50411 Tartu, Estonia. E-mail: kersti.jagomagi@ut.ee

\section{Introduction}

The gold standard method of measuring blood pressure is invasive intra-arterial monitoring. Since invasive monitoring of arterial blood pressure is not feasible in many clinical and experimental settings, the use of non-invasive continuous blood pressure monitoring devices is often mandatory. The following BP monitoring devices can offer non-invasive alternatives to arterial cannulation: Finapres, Finometer, Portapres and TaskForce finger monitors, Colin tonometer, T-line Tensymeter and Vasotrac near-continuous device. The Finapres system, which uses the vascular unloading technique of Penaz together with the Physiocal criteria of Wesseling, is the most often used technique for noninvasive and continuous estimates of blood pressure (Wesseling 1995, Imholz et al. 1998, Birch 2007, Krontorádová et al. 2008, Honzíková and Fišer 2008, Goswami et al. 2008).

In addition, some experimental oscillometric monitors have also been developed to obtain beat-to-beat values of the finger blood pressure (Reeben and Epler 1983, Raamat et al. 2008). In our previous studies we have compared the performance of the experimental oscillometric device UT9201 and Finapres (Portapres) and have found a good agreement between the devices, except the episodes with intensive peripheral vasoconstriction (Jagomägi et al. 1996, Raamat et al. 2000, 2006). Under these conditions the volume-clamp instruments were found to underestimate blood pressure. Similar artifacts of Finapres have been reported by other researchers (Birch 2007).

The Vasotrac APM205A NIBP monitor by 
Medwave (Minneapolis; MN, U. S. A.) measures blood pressure continually by providing one beat of the pressure waveform for approximately every 15 heartbeats. The Vasotrac monitor has been compared to both a radial artery catheter (Belani et al. 1999a, 1999b, Cua et al. 2005, McCann et al. 2005, Hager et al. 2008) and armcuff BP (Thomas et al. 2004, 2005) in various settings and has been found to have in general a good agreement with them. However, it has been found that during liver transplantation when faster changes in BP exist, the readings obtained by the Vasotrac and the direct arterial blood pressure measurements do not agree correctly (Findlay et al. 2006). It is our opinion that the problem of synchronizing of the Vasotrac and the intra-arterial pressure signals of different temporal resolution has not received sufficient attention in the literature and may be partially responsible for the appearance of the disagreement if faster BP changes are compared. Further comparisons of the Vasotrac with faster BP measuring methods should be done.

The objective of this study was to compare the Vasotrac mean blood pressure readings on the radial artery (MAPrad) with the readings obtained by the differential oscillometric monitor, especially designed for the measurement of beat-to-beat mean arterial pressure in finger arteries (MAPfin). Attention was paid to appropriate signal conditioning before comparison of the two pressure signals.

\section{Methods}

\section{Subjects}

The subjects were 14 students, eight females and six males, aged from 19 to 24 years. They had no history of vascular disease and gave their informed consent to participate in the study. The study was approved by the Ethics Committee of the University of Tartu.

The mean subject height and weight were $175 \pm 8.4 \mathrm{~cm}$ and $71.4 \pm 17.8 \mathrm{~kg}$, respectively.

\section{Study protocol}

The subject rested for $10 \mathrm{~min}$ in the supine position, after that blood pressure in both brachial arteries was measured. If the blood pressure differed by more than $5 \mathrm{~mm} \mathrm{Hg}$, the subject was not included in the study.

During the following 8-min data collection period the volunteers lay still and breathed normally; physical and mental stimulations were reduced to a minimum to avoid sympathetic activation, which is known to modulate the resistance of smaller arteries, thus causing variance in the radial-to-finger gradient. Room temperature ranged between 21 and $24^{\circ} \mathrm{C}$.

\section{Finger mean arterial pressure}

MAPfin was measured by the UT9201 physiograph, University of Tartu, Estonia. This instrument applies the oscillometric method for measuring beat-to-beat finger arterial pressure and needs a servosystem to control the counter pressure in the finger cuff (Reeben and Epler 1983, Raamat et al. 2008). In the modified oscillometric instrument the cuff pressure level is kept constant during the systolic part of every cardiac cycle (measuring) and is changed during the diastolic part of the cycle (regulating). By modulating the counter pressure level according to the criterion of getting maximum volume oscillations in the cuff in every cardiac cycle, the counter pressure in the cuff is kept equal to the MAPfin. For a higher reliability the control system is made differential, and it operates with two cuffs on adjacent fingers with pressures shifted from the mean pressure value in both directions for a constant difference. In this 'differential' version the principle of maximum oscillations becomes the principle of the equality of the amplitudes of simultaneous volume oscillations in the two adjacent finger cuffs.

Two cuffs of the UT9201 instrument were attached to the middle and ring fingers of the right hand. Special attention was paid to a proper attachment of finger cuffs to avoid tight or loose fixation.

\section{Radial mean arterial pressure}

Radial mean arterial pressure (MAPrad) was obtained by means of the Vasotrac APM205A NIBP monitor. After a successful self-calibration check the Vasotrac sensor was placed over the left radial artery in the manner described by the manufacturer. Approximately 12 pulse cycles are required for each BP determination. The Vasotrac compresses the sensor over the radial artery at the distal edge of the radius bone. During this process the Vasotrac is looking for the point of maximum energy transfer, i.e. derivation of the maximum pressure pulse signal with minimal sensor pressure over the radial pulse (Belani et al. 1999a). Data were collected with the Vasotrac in continuous mode.

The sensors of both BP measuring devices were carefully kept at the heart level to prevent hydrostatic pressure differences.

To detect single rapid and deep inspirations, 
which result in arteriolar vasoconstriction, we used an elastic plastic tube, wrapped around the chest. Small volume changes of the tube, induced by respiratory volume changes of the chest, were detected by a highsensitivity manometer.

A single investigator placed all the units on the subjects to ensure uniformity.

\section{Signal processing and data analysis}

Simultaneous recordings of MAPrad, MAPfin and respiratory trace were digitized at a sample rate of $100 \mathrm{~Hz}$ and transferred to a personal computer with the custom-made software for subsequent analysis.

In the Vasotrac monitor the number of heart beats used for analysis is approximately 3 to 4 of a 12- to 15-heart beat period (Belani et al. 1999a, 1999b). In the differential oscillometric device the mean blood pressure values are measured during every cardiac cycle and then displayed (Reeben and Epler 1983). Thus, for comparison with the Vasotrac, the data from the oscillometric device should be averaged over the matched consecutive beats. To establish an appropriate match, in preceding experiments we compared each Vasotrac MAP reading with three different sets of finger MAP readings:

i) With a single MAPfin value obtained simultaneously with the updated MAPrad value, $r=0.63$ $(\mathrm{p}<0.02)$;

ii) With MAPfin value averaged over five consecutive heart beats just before updating of the
MAPrad value, $r=0.79(\mathrm{p}<0.001)$;

iii) With MAPfin value averaged over first three heart beats from the set of five consecutive heart beats just before updating of the MAPrad value, $r=0.68$ $(\mathrm{p}<0.01)$.

As the best correlation between MAPrad and MAPfin was found in case ii), this method of signal conditioning was applied in our further study.

Because there can be varying numbers of paired measurements among subjects, the differences between the two methods were determined separately for each person and then averaged across all the subjects to avoid weighting some subjects more than others.

To test the hypotheses a level of significance of 0.05 was applied. Data are expressed as mean \pm SD.

\section{Results}

Data were obtained in 14 healthy persons; a total of 383 pairs of mean blood pressure values from Vasotrac (MAPrad) and finger oscillometric device (MAPfin) were analyzed. Figure 1 is an example of an original recording in one individual. It demonstrates that the proximal (radial) and distal (finger) mean pressures follow each other quite well, with the radial pressure being systematically higher than the digital pressure. The respiration signal (r) demonstrates only one quite deep inspiration (at $390 \mathrm{~s}$ ), which could influence the vasomotor tone.
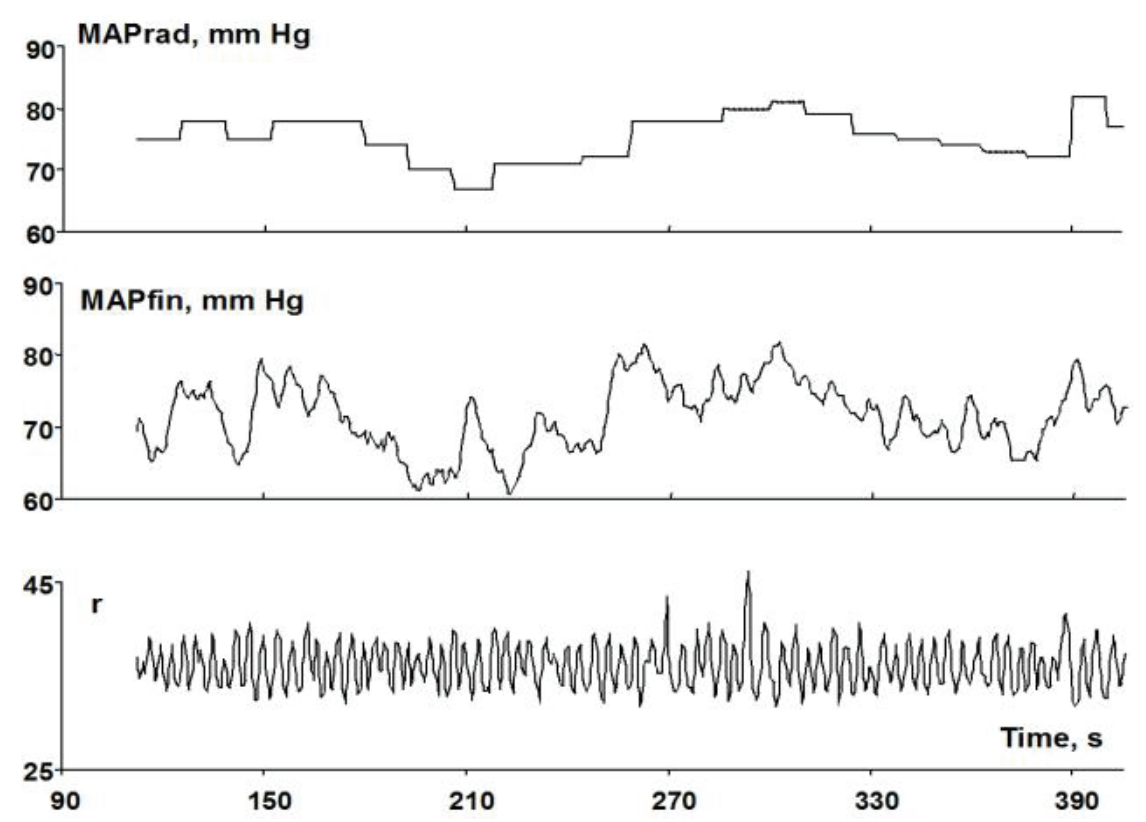

Fig. 1. Example of original recording in one subject. Vasotrac mean blood pressure (MAPrad), oscillometric mean blood pressure (MAPfin) and respiration signal ( $r$ ) are shown. Pressure signals are in $\mathrm{mm} \mathrm{Hg}$, respiration signal is in arbitrary units (au). The respiration signal reflects the phase of respiration (upwards inspiration, downwards - expiration). 


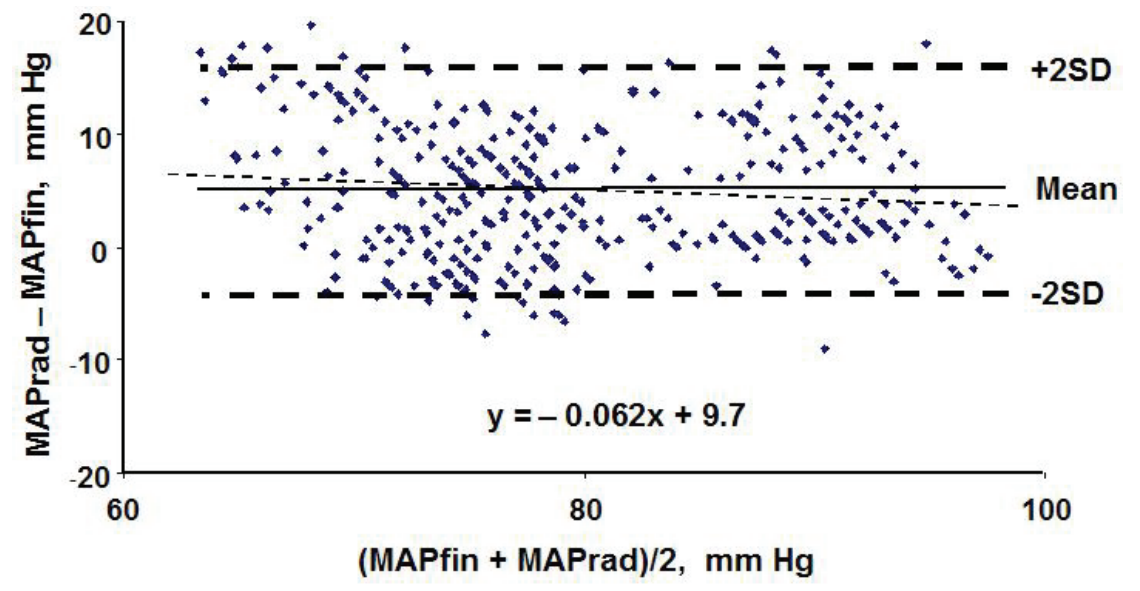

Fig. 2. The Bland-Altman plot for radial mean arterial pressure (MAPrad) and finger mean arterial pressure (MAPfin). Differences between MAPrad and MAPfin are plotted against the average of MAPrad and MAPfin values. The horizontal bold dashed lines represent the $95 \%$ confidence intervals $( \pm 2 \mathrm{SD}$ from the mean difference). The regular dashed line represents an overall linear regression.

Table 1. Mean blood pressure measurement in 14 individuals.

\begin{tabular}{cccc}
\hline Subject & $\begin{array}{c}\text { MAPrad } \\
(\mathbf{m m} \text { Hg) }\end{array}$ & $\begin{array}{c}\text { MAPfin } \\
(\mathbf{m m} \text { Hg) }\end{array}$ & $\begin{array}{c}\text { MAPrad - MAPfin } \\
(\mathbf{m m} \text { Hg) }\end{array}$ \\
\hline 1 & 75.2 & 76.9 & -1.7 \\
2 & 94.4 & 82.7 & 11.7 \\
3 & 93.5 & 93.2 & 0.3 \\
4 & 75.7 & 60.4 & 15.3 \\
5 & 92.4 & 90.4 & 2.0 \\
6 & 75.1 & 71.7 & 3.4 \\
7 & 95.4 & 85.2 & 10.2 \\
8 & 86.3 & 85.9 & 0.4 \\
9 & 83.5 & 76.1 & 7.4 \\
10 & 76.2 & 75.4 & 0.8 \\
11 & 81.2 & 70.9 & 10.3 \\
12 & 79.5 & 74.2 & 5.3 \\
13 & 72.7 & 75.4 & -2.7 \\
14 & 73.9 & 69.4 & 4.5 \\
\hline \multirow{2}{*}{ Average } & 82.5 & 77.7 & 4.8 \\
SD & 8.4 & 8.9 & 5.5 \\
\hline
\end{tabular}

MAP readings were compared graphically by using the Bland-Altman plot (Fig. 2). The Bland-Altman plot provides a visual representation of the data and allows each of the 383 data points to be plotted. Based on all 383 paired measurements, without considering individual persons separately, the Vasotrac measurements for MAP (mean $\pm \mathrm{SD}$ ) were $4.8 \pm 6.0 \mathrm{~mm} \mathrm{Hg}$ higher than MAP values obtained by the oscillometric device.

When considering the individual persons separately and then averaging the mean pressures and their differences for the group of 14 subjects together, we observed the MAP values and differences between the
Vasotrac and differential oscillometric device (Table 1).

The Pearson correlation between the MAPrad and MAPfin values for the whole group was $r=0.79$ $(p<0.001)$. Individual correlations between the two mean arterial blood pressure values ranged between 0.50 and 0.91 .

\section{Discussion}

The main finding of this study is that the MAP values measured by the Vasotrac at the radial artery were $4.8 \pm 6.0 \mathrm{~mm} \mathrm{Hg}$ higher than values measured on fingers 
by the differential oscillometric device.

According to the published data, the finger mean pressure may be some 4-15 $\mathrm{mm} \mathrm{Hg}$ less than the brachial values (Bogert and van Lieshout 2005, Guelen et al. 2008). The pressure difference between the radial and finger arteries is approximately of the same magnitude. Thus, we can consider that the radial to finger difference of $4.8 \mathrm{~mm} \mathrm{Hg}$ measured in our study at rest in supine position is consistent with previous investigations and the Vasotrac monitor adequately tracked relatively fast spontaneous MAP changes of the radial artery in our experiments (Fig. 1).

As far as the signal matching is concerned, different sets of BP values have been used for comparison of two pressure signals in published articles. In most cases the authors have reported that the Vasotrac beat used to calculate BP was synchronized with the corresponding radial artery beat by applying a computer (Belani et al. 1999b, McCann et al. 2005, Findlay et al. 2006). However, it remains unclear how the heart beat utilized by the Vasotrac was selected.

As the Vasotrac system uses several beats to calculate BP algorithmically, it is hard to understand the comparison of $\mathrm{BP}$ on one beat basis.

The methodology of synchronization applied in the present study compared each MAPrad value with MAPfin value averaged over five consecutive heart beats preceding the corresponding MAPrad exposure. This method of signal conditioning was found optimal for the subjects in our experiments.

We consistently used the Vasotrac device on the left arm and the differential oscillometric device on the right one. Any side-to-side difference in the arterial blood pressure time course would be recorded as a difference between the two devices. As the left upper arm versus the right upper arm BP difference was checked in each of our young healthy volunteers before the experiment, the side to side difference is very unlikely to be the explanation for the differences that we have observed.

\section{Conclusions}

MAP values measured at the radial artery at rest were $4.8 \pm 6.0 \mathrm{~mm} \mathrm{Hg}$ higher than those measured on fingers, which can be explained by different measurement sites. The Vasotrac monitor can be considered to adequately track relatively fast MAP changes of the radial artery in our experiments. Attention should be paid to a proper signal conditioning before the comparison of results obtained by different devices.

\section{Conflict of Interest}

There is no conflict of interest.

\section{Acknowledgements}

This work was supported by grant 6947 from the Estonian Science Foundation.

\section{References}

BELANI K, OZAKI M, HYNSON J, HARTMANN T, REYFORD H, MARTINO JM, POLIAC M, MILLER R: A new non-invasive method to measure blood pressure: results of a multicenter trial. Anesthesiology 91: 686692, 1999a.

BELANI KG, BUCKLEY JJ, POLIAC MO: Accuracy of radial artery blood pressure determination with the Vasotrac. Can J Anaesth 46: 488-496, 1999b.

BIRCH AA: Sympathetic peripheral vasoconstriction may be measured using an artifact of the Finapres volume clamp technique. Blood Press Monit 12: 315-319, 2007.

BOGERT LW, VAN LIESHOUT JJ: Non-invasive pulsatile arterial pressure and stroke volume changes from the human finger. Exp Physiol 90: 437-446, 2005.

CUA CL, THOMAS K, ZURAKOWSKI D, LAUSSEN PC: A comparison of the Vasotrac with invasive arterial blood pressure monitoring in children after pediatric cardiac surgery. Anesth Analg 100: 289-294, 2005.

FINDLAY JY, GALI B, KEEGAN MT, BURKLE CM, PLEVAK DJ: Vasotrac arterial blood pressure and direct arterial blood pressure monitoring during liver transplantation. Anesth Analg 102: 690-693, 2006.

HAGER H, MANDADI G, PULLEY D, EAGON JC, MASCHA E, NUTTER B, KURZ A: A comparison of noninvasive blood pressure measurement on the wrist with invasive arterial blood pressure monitoring in patients undergoing bariatric surgery. Obes Surg 19: 717-724, 2009. 
GOSWAMI N, GRASSER E, ROESSLER A, SCHNEDITZ D, HINGHOFER-SZALKAY H: The cardiovascular response to lower body negative pressure in humans depends on seal location. Physiol Res 58: 311-318, 2009.

GUELEN I, WESTERHOF BE, VAN DER SAR GL, VAN MONTFRANS GA, KIEMENEIJ F, WESSELING KH, BOS WJ: Validation of brachial artery pressure reconstruction from finger arterial pressure. J Hypertens 26: 13211327, 2008.

HONZÍKOVÁ N, FIŠER B: Baroreflex sensitivity and essential hypertension in adolescents. Physiol Res 58: 605-612, 2009.

IMHOLZ BP, WIELING W, VAN MONTFRANS GA, WESSELING KH: Fifteen years experience with finger arterial pressure monitoring: assessment of the technology. Cardiovasc Res 38: 605-616, 1998.

JAGOMÄGI K, TALTS J, RAAMAT R, LÄNSIMIES E: Continuous non-invasive measurement of mean blood pressure in fingers by volume-clamp and differential oscillometric method. Clin Physiol 16: 551-560, 1996.

KRONTORÁDOVÁ K, HONZÍKOVÁ N, FIŠER B, NOVÁKOVÁ Z, ZÁVODNÁ E, HRSTKOVÁ H, HONZÍK P: Overweight and decreased baroreflex sensitivity as independent risk factors for hypertension in children, adolescents, and young adults. Physiol Res 57: 385-391, 2008.

MCCANN ME, HILL D, THOMAS KC, ZURAKOWSKI D, LAUSSEN PC: A comparison of radial artery blood pressure determination between the Vasotrac device and invasive arterial blood pressure monitoring in adolescents undergoing scoliosis surgery. Anesth Analg 101: 78-85, 2005.

RAAMAT R, JAGOMÄGI K, TALTS J: Different responses of Finapres and the oscillometric finger blood pressure monitor during intensive vasomotion. J Med Eng Technol 24: 95-101, 2000.

RAAMAT R, TALTS J, JAGOMÄGI K, KIVASTIK J, LÄNSIMIES E, JURVELIN J: Simultaneous application of differential servo-oscillometry and volume-clamp plethysmography for continuous non-invasive recording of the finger blood pressure response to a hand postural change. J Med Eng Technol 30: 139-144, 2006.

RAAMAT R, JAGOMÄGI K, TALTS J: Continuous recording of the oscillometric mean arterial pressure by the differential servo system with two photoplethysmographic sensors. Proceedings of the 8th IEEE International Conference BioInformatics and BioEngineering, BIBE 2008, 1-4, Athens, 2008.

REEBEN V, EPLER M: Indirect continuous measurement of mean arterial pressure In: Advances in Cardiovascular Physics, vol. 5, Cardiovascular Engineering. Part II: Monitoring. GHISTA DN (ed), Karger, Basel, 1983, pp 90-118.

THOMAS SH, WINSOR GR, PANG PS, DRISCOLL KA, PARRY BA: Use of a radial artery compression device for noninvasive, near-continuous blood pressure monitoring in the ED. Am J Emerg Med 22: 474-478, 2004.

THOMAS SH, WINSOR G, PANG P, WEDEL SK, PARRY B: Near-continuous, noninvasive blood pressure monitoring in the out-of-hospital setting. Prehosp Emerg Care 9: 68-72, 2005.

WESSELING KH: A century of non-invasive arterial pressure measurement: from Marey to Penaz and Finapres. Homeostasis 36: 50-66, 1995. 